Supporting information for

\title{
Blood Pyrrole-DNA Adducts Define the Early Tumorigenic Risk in Patients with Pyrrolizidine Alkaloid-Induced Liver Injury
}

Yisheng He, Wei Zhang, Jiang Ma, Qingsu Xia, Zijing Song, Lin Zhu, Chunyuan

Zhang, Jia Liu, Yang Ye, Peter P. Fu, Yuzheng Zhuge, Ge Lin

\section{Correspondence}

Ge Lin (linge@cuhk.edu.hk); Yuzheng Zhuge (yuzheng9111963@aliyun.com) 


\section{Supporting methods}

\section{Caution}

Retrorsine, riddelliine, monocrotaline, and clivorine are carcinogenic in laboratory animals. They should be handled with extreme care, using proper personal protective equipment and a well-ventilated hood.

\section{Rat model}

Sprague Dawley rats were maintained at our animal facility according to the guidelines of the Animal Experimental Ethics Committee, The Chinese University of Hong Kong. Rats were kept in groups ( $n=3$ per cage) under standard housing conditions with 12-hour light-dark cycle and free access to food pellets and drinking water.

\section{Pyrrole-DNA adducts and analyses using enzymatic hydrolysis method}

The concentration and purity of the extracted DNA were determined using NanoDrop 2000c spectrophotometer (Thermo Fisher Scientific, San Jose, CA, USA). To quantify individual pyrrole-dA adducts and pyrrole-dG adducts, DNA samples (100 $\mu \mathrm{g})$ were enzymatically hydrolyzed to nucleotides via micrococcal nuclease, phosphodiesterase, and nuclease P1 (Sigma-Aldrich, St. Louis, MO, USA). The hydrolyzed products were subject to liquid chromatography coupled with tandem mass spectrometry (LC-MS/MS) analysis.

The quantitation of PDAs performed using Agilent 1290 Infinity ultra-high-performance liquid chromatography system equipped with Agilent 6460 Mass Spectrometer detector (Agilent Technologies, Santa Clara, CA, USA). Chromatographic separation was performed using an Acquity BEH $(2.1 \times 100$ mm, 1.7 $\mu \mathrm{m}$ ) C18 column (Waters, Milford, MA, USA). The mobile phase consisted of deionized water (A) and acetonitrile (B). A gradient elution procedure was used: 0-0.5 
min, $0 \%$ B; 0.5-20 min, 0-15 \% B; 20-22 min, 15-18 \%; 22-24 min, 18-95 \%. The flow rate was $0.3 \mathrm{~mL} / \mathrm{min}$, and the column temperature was maintained at $45^{\circ} \mathrm{C}$. The mass spectrometer was operated on multiple reaction monitoring and positive ion mode. The characteristic ion pairs for pyrrole-dG adducts were $m / z 403 \rightarrow 269$, and ion pairs for pyrrole-dA adducts were $\mathrm{m} / \mathrm{z} 387 \rightarrow 253$.

\section{Pyrrole-DNA adducts analyses using chemical derivatization method}

In order to release pyrrole moiety from the pyrrole-DNA adducts, the precipitated DNA pellets were mixed with 5 volumes of $2 \%$ acidic silver nitrate ethanol solution containing $20 \mathrm{mg} / \mathrm{mL}$ silver nitrate and 5\% trifluoroacetic acid. The mixture was shaken for $30 \mathrm{~min}$ and then centrifuged at $15,000 \mathrm{~g}$ for $10 \mathrm{~min}$. The supernatant $(80 \mu \mathrm{L})$ was reacted with $20 \mu \mathrm{L}$ of 4 -(dimethylamino) benzaldehyde $(20 \mathrm{mg} / \mathrm{mL})$ in ethanol containing $1 \%$ perchloric acid at $55^{\circ} \mathrm{C}$ for $10 \mathrm{~min}$. The resultant pyrrolic derivative, 7,9-diethoxy-DHP-4-(dimethylamino)benzaldehyde, is the common derivative of all possible pyrrole-DNA adducts. The quantitative analysis on the derivative was performed by LC-MS/MS, using Agilent 1290 Infinity ultra-high-performance liquid chromatography system equipped with Agilent 6460 Mass Spectrometer detector. Chromatographic separation was performed using an Acquity BEH (2.1×100 mm, 1.7 $\mu \mathrm{m})$ C18 column. The mobile phase consisted of water (A) and acetonitrile (B), both containing $0.1 \%$ formic acid. Each sample was injected with $3 \mu \mathrm{L}$ of volume into the column and eluted $(0.3 \mathrm{~mL} / \mathrm{min})$ with an optimized linear gradient: $0-5 \mathrm{~min}, 65-5 \%$ B; $5-5.5 \mathrm{~min}, 5 \% \mathrm{~B} ; 5.5-6 \mathrm{~min}, 5-65 \% \mathrm{~B}$. The flow rate was $0.3 \mathrm{~mL} / \mathrm{min}$, and the column temperature was maintained at $25^{\circ} \mathrm{C}$. Analytes were detected by mass spectrometer under the multiple reaction monitoring and positive ion mode, with characteristic ion pairs $\mathrm{m} / \mathrm{z} 341.2>252.2$ for the derivative analyte. The fragmentor and collision energy were $185 \mathrm{~V}$ and $33 \mathrm{~V}$. 


\section{Supporting figures}
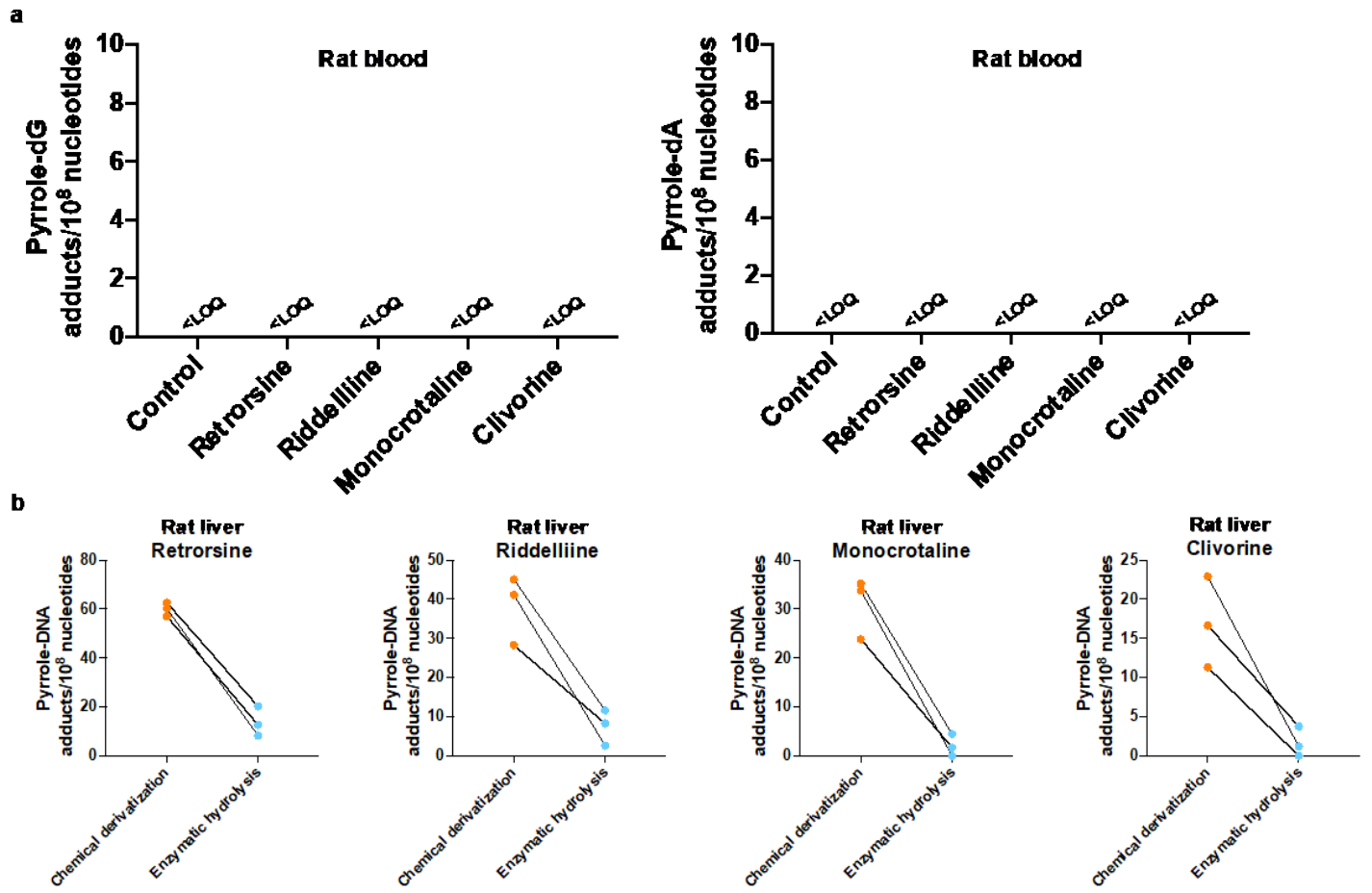

Figure S1. (a) Enzymatic hydrolysis determination of pyrrole-dG adducts and pyrrole-dA adducts in blood samples from rats exposed to four different PAs (0.2 $\mathrm{mmol} / \mathrm{kg}, n=3$ per PA-treated rat group), confirming the infeasibility of the conventional method for detecting blood PDA. $<$ LOQ, below limit of quantitation. (b) Comparison of PDAs levels measured by chemical derivatization method and enzymatic hydrolysis method in PA-exposed rat livers $(n=3)$. The PDAs measured by enzymatic hydrolysis method are the sum of pyrrole-dG adducts and pyrrole-dA adducts. The levels of hepatic PDAs detected by the derivatization method were at least 5-fold higher than that detected by the enzymatic hydrolysis method. 

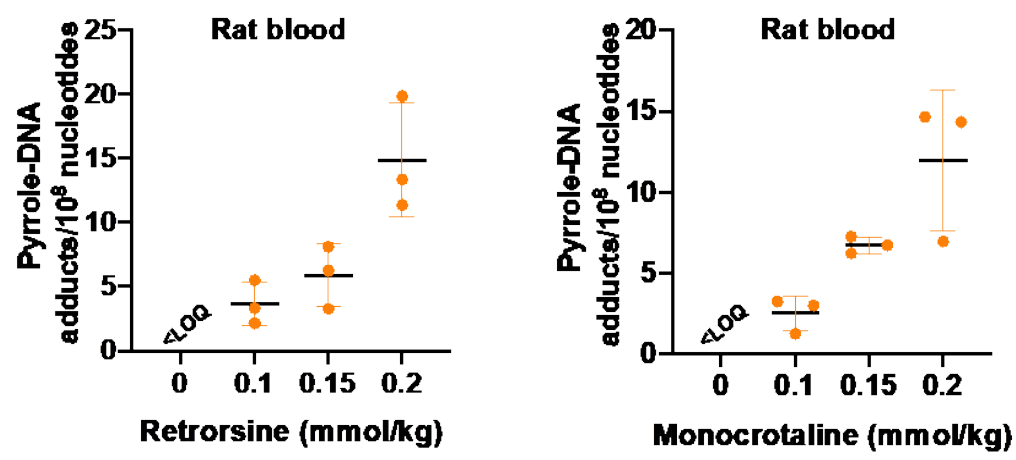

Figure S2. Rats $(n=3)$ were orally administered with two different PAs (retrorsine and monocrotaline) in different doses $(0,0.1,0.15$, and $0.2 \mathrm{mmol} / \mathrm{kg}$ ), and blood samples were collected at 48 hours after PA treatments. The blood PDA levels, measured by the chemical derivatization method, show a dose dependent manner in both retrorsine-treated and monocrotaline-treated rats. $<\mathrm{LOQ}$, below limit of quantitation. 

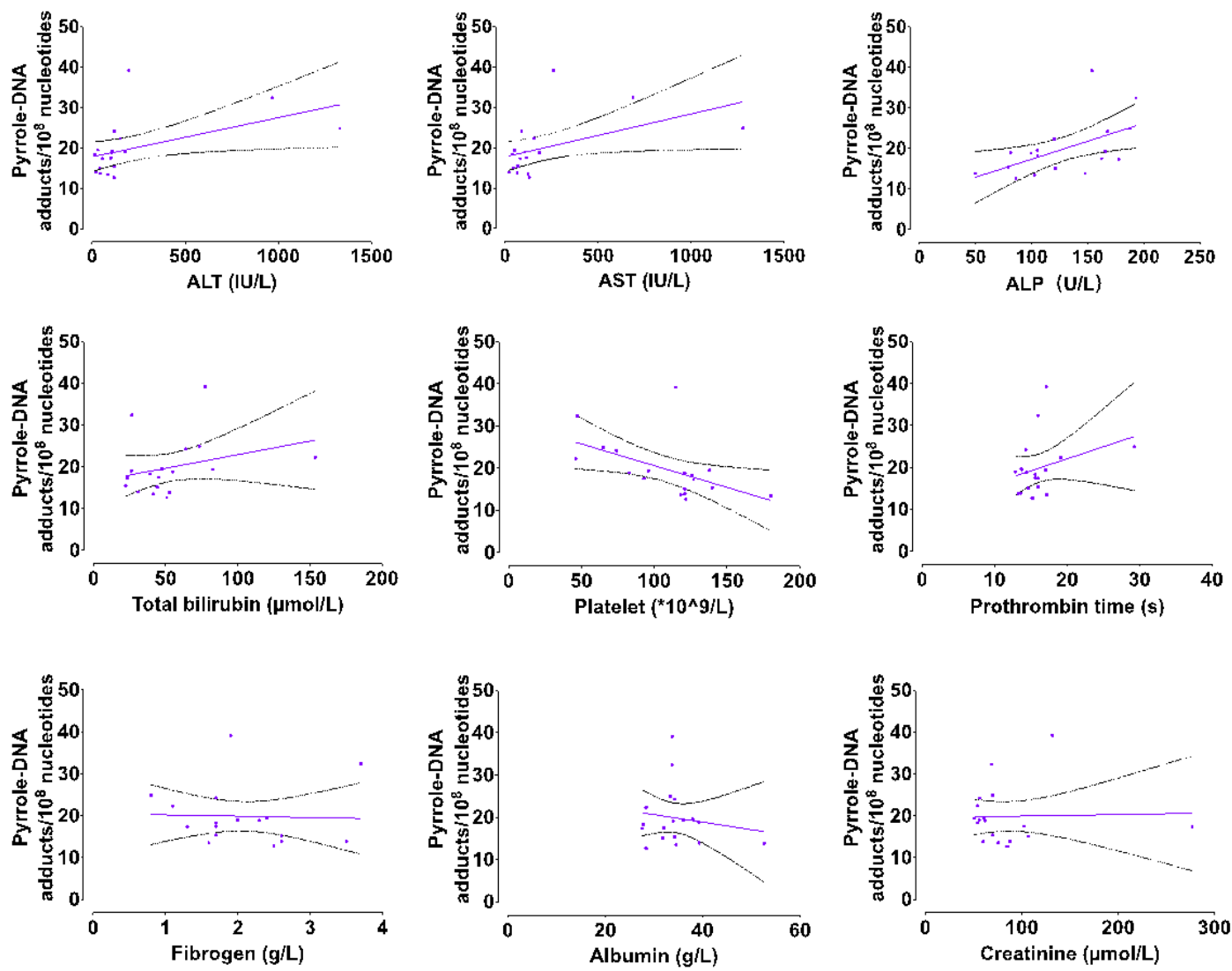

Figure S3. Correlation tests between PDA levels and clinical test indices involving general liver damage (ALT, AST, AP), cholestasis (total bilirubin), blood coagulation (platelet, prothrombin time, fibrogen), kidney function (creatinine), and liver function (albumin). The correlation test results are provided in Table S3. ALT, alanine aminotransferase; AST, aspartate aminotransferase; ALP, alkaline phosphatase. 


\section{Supporting tables}

Table S1. Demographic information (gender, intake of PA-containing herbal remedy) and clinical features (liver injury manifestation, RUCAM score, treatment) of PA-ILI ${ }^{a}$ patients $(n=18)$. Data are presented as the number of patients or percentage as appropriate.

\begin{tabular}{|c|c|c|c|}
\hline & & Number of patients & Percentage \\
\hline \multirow[t]{2}{*}{ Gender } & Male & 10 & $56 \%$ \\
\hline & Female & 8 & $44 \%$ \\
\hline \multirow{3}{*}{$\begin{array}{l}\text { Clinical types of } \\
\text { liver injury }\end{array}$} & Cholestatic injury & 6 & $33 \%$ \\
\hline & Hepatocellular injury & 6 & $33 \%$ \\
\hline & Mixed injury & 6 & $33 \%$ \\
\hline \multirow{5}{*}{$\begin{array}{l}\text { Clinical } \\
\text { manifestations }\end{array}$} & Ascites & 18 & $100 \%$ \\
\hline & Abdominal distension & 17 & $94 \%$ \\
\hline & Jaundice & 11 & $61 \%$ \\
\hline & Hepatomegaly & 10 & $56 \%$ \\
\hline & Right quadrant pain & 5 & $28 \%$ \\
\hline \multirow{2}{*}{ RUCAM $^{b}$ score } & $6-8$ & 11 & $61 \%$ \\
\hline & $9-10$ & 7 & $39 \%$ \\
\hline \multirow{3}{*}{$\begin{array}{l}\text { Dosage form of } \\
\text { Gynura japonica }\end{array}$} & Decoction & 11 & $61 \%$ \\
\hline & Herbal wine & 4 & $22 \%$ \\
\hline & Ground powder & 3 & $17 \%$ \\
\hline \multirow{5}{*}{$\begin{array}{l}\text { Intake duration of } \\
\text { Gynura japonica }\end{array}$} & $\leq 7$ days & 1 & $6 \%$ \\
\hline & 10-20 days & 8 & $44 \%$ \\
\hline & $1-2$ months & 5 & $28 \%$ \\
\hline & $\geq 3$ months & 3 & $17 \%$ \\
\hline & Unknown & 1 & $6 \%$ \\
\hline \multirow[t]{3}{*}{ Treatment } & Anticoagulation & 18 & $100 \%$ \\
\hline & TIPS & 12 & $67 \%$ \\
\hline & & Median and range & Reference range \\
\hline \multirow[t]{6}{*}{ Laboratory test } & $\mathrm{ALT}^{d}(\mathrm{U} / \mathrm{L})$ & $106.95(29.3-1329.3)$ & 6-40 U/L \\
\hline & $\mathrm{AST}^{e}(\mathrm{U} / \mathrm{L})$ & 98.25 (22.6-1278.4) & $6-40 \mathrm{U} / \mathrm{L}$ \\
\hline & Total bilirubin $(\mu \mathrm{mol} / \mathrm{L})$ & $46.30(23.3-153.6)$ & $5-17.5 \mu \mathrm{mol} / \mathrm{L}$ \\
\hline & $\operatorname{ALPf}_{(\mathrm{U} / \mathrm{L})}$ & 120.85 (49.6-193.2) & $11-50 \mathrm{U} / \mathrm{L}$ \\
\hline & Albumin (g/L) & $33.80(27.7-39.3)$ & $35-50 \mathrm{~g} / \mathrm{L}$ \\
\hline & Prothrombin time (s) & $15.60(12.9-29.3)$ & $10-15 \mathrm{~s}$ \\
\hline
\end{tabular}


${ }^{a}$ PA-ILI: PA-induced liver injury. ${ }^{b}$ RUCAM: the Roussel Uclaf Causality Assessment Method. 'TIPS: transjugular intrahepatic portosystemic stentshunt. ${ }^{d}$ ALT: alanine aminotransferase. ${ }^{e}$ AST: aspartate aminotransferase. ${ }^{f} \mathrm{ALP}$ : alkaline phosphatase. 
Table S2. Demographic information (age, gender, intake of PA-containing herbal remedy), biochemical assay (ALT ${ }^{a}, \mathrm{ALP}^{b}$ ), and PA biomarker assay $\left(\mathrm{PDAs}^{c}, \mathrm{PPAs}^{d}\right)$ of individual PA-ILI ${ }^{e}$ patients $(n=18)$.

\begin{tabular}{|c|c|c|c|c|c|c|c|c|}
\hline Patient ID & Sex & Gender & Herb ingested & Intake dose/period & $\operatorname{ALT}(\mathbf{U} / \mathbf{L})$ & $\operatorname{ALP}(\mathbf{U} / \mathbf{L})$ & PDAs $/ 10 \wedge 8$ nucleotides & PPAs (nM) \\
\hline 1 & Male & 72 & decoction & 10 days & 54.4 & 177.6 & 17.3 & 4 \\
\hline 2 & Male & 54 & powder drunk with sugar water & 1 month & 118.4 & 86 & 12.7 & 19.7 \\
\hline 3 & Female & 70 & herbal wine & 50 days & 83.4 & 102.3 & 13.5 & 7.4 \\
\hline 4 & Female & 80 & herbal wine & $50-100 \mathrm{~mL} /$ day, 3 months & 29.3 & 105.5 & 19.6 & 14.1 \\
\hline 5 & Female & 65 & stems used for decoction & 6 months & 105.9 & 99.5 & 18.9 & 14.3 \\
\hline 6 & Male & 67 & decoction & 10 days & 1329.3 & 187.9 & 24.9 & 39.6 \\
\hline 7 & Male & 43 & leaves for decoction & 20 days & 118.8 & 79.2 & 15.4 & 31.8 \\
\hline 8 & Male & 52 & decoction & 2 months & 150.6 & 120.3 & 22.3 & 29.7 \\
\hline 9 & Male & 55 & powder & 3-4 g/days & 100.6 & 162.3 & 17.5 & 14.9 \\
\hline 10 & Female & 71 & decoction & 10 days & 177 & 81.3 & 19.0 & 57.4 \\
\hline
\end{tabular}




\begin{tabular}{|c|c|c|c|c|c|c|c|c|}
\hline 11 & Female & 77 & herbal wine & 0.5 month & 120.4 & 167.7 & 24.2 & 202.1 \\
\hline 12 & Female & 52 & roots cut to pieces & 2-3 pieces/day,1 month & 44.8 & 147.7 & 13.8 & 46.2 \\
\hline 13 & Male & 63 & decoction & 15 days & 967 & 193.2 & 32.4 & 195.4 \\
\hline 14 & Male & 68 & decoction & 15 days & 198.4 & 153.7 & 39.2 & 302.2 \\
\hline 15 & Female & 58 & roots and leaves for decoction & 10 days & 108 & 165.8 & 19.3 & 45.1 \\
\hline 16 & Male & 69 & decoction & 1 month & 42.4 & 121.4 & 15.1 & 10.4 \\
\hline 17 & Male & 72 & cut to slices for herbal wine & $100 \mathrm{ml} /$ day, 1 month & 19.6 & 49.6 & 13.9 & 15.9 \\
\hline 18 & Female & 68 & decoction & 20 years & 15.9 & 105.4 & 18.3 & 78.8 \\
\hline
\end{tabular}

${ }^{a}$ ALT: serum alanine transaminase activity. ${ }^{b}$ ALP: serum alkaline phosphatase activity. ${ }^{c}$ PDAs, blood pyrrole-DNA adducts. ${ }^{d}$ PPAs, blood pyrrole-protein adducts. PPA data of the 18 PA-ILI patients have been published. ${ }^{1}{ }^{e}$ PA-ILI, pyrrolizidine alkaloid-induced liver injury. 
Table S3. Correlation test of PDA levels and liver injury biomarkers in PA-ILI ${ }^{a}$ patients $(n=18)$.

\begin{tabular}{lccc} 
& Spearman $\mathbf{~}$ & 95\% Confidence interval & P value \\
\hline Age & 0.1364 & $-0.3659-0.5772$ & 0.5893 \\
ALT $^{b}$ & 0.6202 & $0.2016-0.8473$ & 0.0060 \\
AST $^{c}$ & 0.4572 & $-0.02728-0.7677$ & 0.0565 \\
ALP $^{d}$ & 0.5562 & $0.1060-0.8172$ & 0.0165 \\
Total bilirubin & 0.4076 & $-0.08802-0.7415$ & 0.0931 \\
Platelet & -0.6443 & $-0.8582--0.2397$ & 0.0039 \\
Prothrombin time & 0.3145 & $-0.1930-0.6893$ & 0.2036 \\
Fibrogen & -0.2035 & $-0.6215-0.3046$ & 0.4179 \\
Albumin & -0.08992 & $-0.5450-0.4060$ & 0.7227 \\
Creatinine & -0.2894 & $-0.6745-0.2195$ & 0.2441 \\
\hline
\end{tabular}

${ }^{a}$ PA-ILI, pyrrolizidine alkaloid-induced liver injury. ${ }^{b} \mathrm{ALT}$ : serum alanine transaminase activity. ${ }^{c}$ AST, serum aspartate transaminase activity. ${ }^{d}$ ALP: serum alkaline phosphatase activity. 


\section{References}

(1) Ma, J.; Zhang, W.; He, Y.; Zhu, L.; Zhang, C.; Liu, J.; Ye, Y.; Zhuge, Y.; Lin, G. Clinical application of pyrrole-hemoglobin adducts as a biomarker of pyrrolizidine alkaloid exposure in humans. Arch Toxicol 2021, 95 (2), 759-765. 